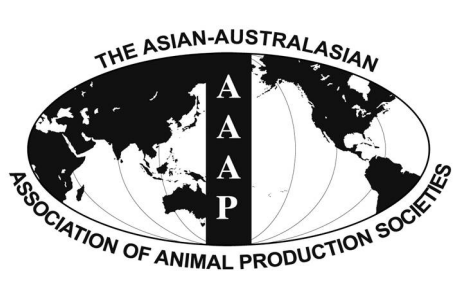

Open Access

Asian Australas. J. Anim. Sci.

Vol. 29, No. 9 : 1345-1352 September 2016

http://dx.doi.org/10.5713/ajas.15.0587

www.ajas.info

pISSN 1011-2367 elSSN 1976-5517

\title{
Comparison of Fecal Microbiota of Mongolian and Thoroughbred Horses by High-throughput Sequencing of the V4 Region of the 16S rRNA Gene
}

\author{
Yiping Zhao, Bei Li, Dongyi Bai, Jinlong Huang, Wunierfu Shiraigo, Lihua Yang, Qinan Zhao, \\ Xiujuan Ren, Jing Wu, Wuyundalai Bao, and Manglai Dugarjaviin* \\ College of Animal Science, Inner Mongolia Agricultural University, \\ Inner Mongolia Mongolian Horse Genetic Resources Protection and Industrial Engineering Laboratory, \\ Hohhot 010018, China
}

\begin{abstract}
The hindgut of horses is an anaerobic fermentative chamber for a complex and dynamic microbial population, which plays a critical role in health and energy requirements. Research on the gut microbiota of Mongolian horses has not been reported until now as far as we know. Mongolian horse is a major local breed in China. We performed high-throughput sequencing of the 16S rRNA genes V4 hypervariable regions from gut fecal material to characterize the gut microbiota of Mongolian horses and compare them to the microbiota in Thoroughbred horses. Fourteen Mongolian and 19 Thoroughbred horses were used in the study. A total of 593,678 sequence reads were obtained from 33 samples analyzed, which were found to belong to 16 phyla and 75 genera. The bacterial community compositions were similar for the two breeds. Firmicutes (56\% in Mongolian horses and 53\% in Thoroughbred horses) and Bacteroidetes (33\% and 32\% respectively) were the most abundant and predominant phyla followed by Spirochaete, Verrucomicrobia, Proteobacteria, and Fibrobacteres. Of these 16 phyla, five (Synergistetes, Planctomycetes, Proteobacteria, TM7, and Chloroflexi) were significantly different $(\mathrm{p}<0.05)$ between the two breeds. At the genus level, Treponema was the most abundant genus $(43 \%$ in Mongolian horses vs $29 \%$ in Thoroughbred horses), followed by Ruminococcus, Roseburia, Pseudobutyrivibrio, and Anaeroplasma, which were detected in higher distribution proportion in Mongolian horses than in Thoroughbred horses. In contrast, Oscillibacter, Fibrobacter, Methanocorpusculum, and Succinivibrio levels were lower in Mongolian horses. Among 75 genera, 30 genera were significantly different $(\mathrm{p}<0.05)$ between the two breeds. We found that the environment was one of very important factors that influenced horse gut microbiota. These findings provide novel information about the gut microbiota of Mongolian horses and a foundation for future investigations of gut bacterial factors that may influence the development and progression of gastrointestinal disease in horses. (Key Words: Fecal Microbiota, 16S rRNA Gene, High-throughput Sequencing, Mongolian Horses)
\end{abstract}

\section{INTRODUCTION}

Animal gut microbiota plays important roles in harvesting nutrientsand extracting energy from the diet, resisting the colonization of pathogens, developing the immune system, and detoxifying toxic compounds in the host. The horse has evolved as a grazing nonruminant herbivore with a specialized gastrointestinal tract anatomy that is known as hindgut fermenters capable of utilizing a wide range of plant species (Costa et al., 2012). The hindgut

\footnotetext{
* Corresponding Author: Manglai Dugarjaviin. Tel: +86-471-

4316151, Fax: +86-471-4309408, E-mail: dmanglai@163.com Submitted Jul. 10, 2015; Revised Nov. 11, 2015; Accepted Dec. 1, 2015
}

fermenters contain a complex microbial digestion uniquely adapted to grazing on high-fibre, low-energy fodder (Dougal et al., 2012), degrade and ferment to short chain fatty acids that in turn can beabsorbed and utilized as energy sources (Blackmore et al., 2013).

Animal microbial communities are affected by external factors such as lifestyle, environment (Ley et al., 2008), dietarypatterns (Dougal et al., 2014), etc. The homeostatic balance in the equine intestinal microbiome is sensitive to factors like gastrointestinal disease and dietary change, including fermentative acidosis, laminitis, and colic (Costa et al., 2012; Steelman et al., 2012). Some reports suggest greater extent of hindgut microbial adaptation in native

Copyright @ 2016 by Asian-Australasian Journal of Animal Sciences This is an open-access article distributed under the terms of the Creative Commons Attribution Non-Commercial License (http://creativecommons.org/licenses/by-nc/3.0/), which permits unrestricted non-commercial use, distribution, and reproduction in any medium, provided the original work is properly cited. 
horses to a low quality winter diet that leads a higher degree of hindgut fermentation (Obitsu et al., 2015). The Mongolian horse originated in the Inner Mongolian grasslands in China. Mongolian grasslands are quite vast from east to west and the ecological and natural conditions are varied. As a result, Mongolian horses are adapted to cold, insufficient food and hardwork and can endure rather rough feeding and management. They show strong vital force and can survive under harsh conditions. However, the Thoroughbred is a famous race horse for its speed in short distance races. They are quite strict for requirements of breeding management and nutrition besides to sufficient amount feed available. Therefore, these two species have very different nutrition, management, and environment.

Studies using direct sequencing methods have revealed a greater diversity of horse microbiota than previously estimated by culture-dependent methods (Gustafsson et al., 1999). Previous were insufficient to fully understand the horse microbial communities. Direct sequencing analysis was used to study the gut microbiota of Irish Thoroughbred race horses in a recent study (O'Donnell et al., 2013), Ponies (Gustafsson et al., 1999) and so on. We examined and compared the composition and variation in the fecal microbiota of two horse breeds, Mongolian horses and Thoroughbred horses, using the high-throughput sequencing.

\section{MATERIALS AND METHODS}

\section{Sample collection}

Protocols used for this experiment were consistent with those approved by the Institutional Animal Care and Use Committee at Inner Mongolia Agricultural University. Fresh fecal samples were collected from 14 healthy Mongolian horses with a mean age of 5.29 years (range, 2 to 12 years, $4 \hat{\sigma}$ and 10 +) and 19 healthy Thoroughbred horses with a mean age of 5.89 years (range, 4 to 7 years, 5 त and 14 ㅇ) in September in the morning. All horses were kept on two different farms in Inner Mongolia of China for at least 12 months and had not experienced any recent changes in diet or housing conditions. Mongolian horses were grazed on the prairie but Thoroughbred horses lived in the stable. Every sample was obtained immediately after defecation, transferred into a separate sterilized container, and stored on ice for transport to the laboratory and stored at $-80^{\circ} \mathrm{C}$.

\section{DNA extraction}

The total genomic DNA was extracted from feces using QIAamp DNA stool mini kit (QIAGEN, Hilden, Germany, cat\#51504) using the manufacture's protocols. The V4 region of $16 \mathrm{~S}$ rRNA gene was polymerase chain reaction (PCR) amplified from microbial genome DNA which was harvested from fecal samples using barcoded fusion primers (forward primers: 5'-AYTGGGYDTAAA GNG-3' and reverse primers: 5'-TACNVGGGTATC TAATCC-3'). The thermal conditions were $98^{\circ} \mathrm{C}$ denaturation for $30 \mathrm{~s}, 27$ cycles of $98^{\circ} \mathrm{C}$ for $30 \mathrm{~s}, 50^{\circ} \mathrm{C}$ for $30 \mathrm{~s}, 72^{\circ} \mathrm{C}$ for $30 \mathrm{~s}$, and a final 5 min elongation step at $72^{\circ} \mathrm{C}$. PCR product was excised from a $0.8 \%$ agarose gel and purified by AxyPrep DNA Gel Extraction Kit (AXYGEN Inc., Union City, CA USA,cat\#AP-GX-500).

\section{Sequencing}

V4 amplicons were sequenced using pair-end method by Illumina Miseq. Sequences were constructed after trimming positions with $<$ Q25 quality score and discarding reads $<50 \mathrm{bp}$, ambiguous bases, homopolymer run exceeds 6 , and having mismatches in primers. Then, these reads were assembled by Flash software (http://www.genomics.jhu.edu/software/FLASH/index.shtml), and the reads which could not assembled were discarded.

\section{Sequence analysis}

The operational taxonomic unit (OTU) assignments were made after clustering the sequences with a similarity cutoff of $97 \%$ by uclust quantitative insights into microbial ecology (QIIME). The longest sequence in each cluster was chosen to be the representative sequence which was annotated by Ribosomal Database Project (RDP)-classifier 2.2 (QIIME).

The alpha diversity analysis (Chao1, ACE, Simpson index, Shannon index, and Coverage) was performed using the summary single command of MOTHUR software (http://www.mothur.org/). $T$ tests were performed by Microsoft excel and all the $\mathrm{p}$ values were calculated by false discovery rate (FDR) using p.adjust package of $R$ (http://stat.ethz.ch/R-manual/R-devel/library/stats/html/p.adjust. $\mathrm{html}$ ) for correcting. FDR $=0.2$ was used as a threshold throughout the analysis.

\section{RESULTS}

\section{Sequencing and classification}

We applied 16S rRNA gene (V4 region) amplicon to determine the fecal microbiota composition of 14 Mongolian and 19 Thoroughbred horses using pair-end method by Illumina Miseq. After quality control, a total of 593,678 reads of V4 16S rRNA sequences were obtained from the 33 samples $(245,733$ for Mongolian horses and

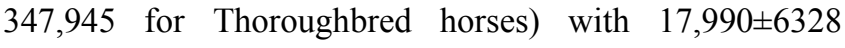
sequence reads per sample $(17,552 \pm 5,941$ and 18,312 $\pm 6,741$, Mongolian and Thoroughbred horses respectively). The average length of sequence reads was $225 \mathrm{bp}$, and they were classified using QIIME into different OTUs based on the identity level at $97 \%$. A total of 91,987 OTUs were obtained and each individual averaged 2,787 OTUs (range $=1,927$ to 4,785 , standard deviation [SD] $=$ 614). The taxon abundance of each sample was generated 


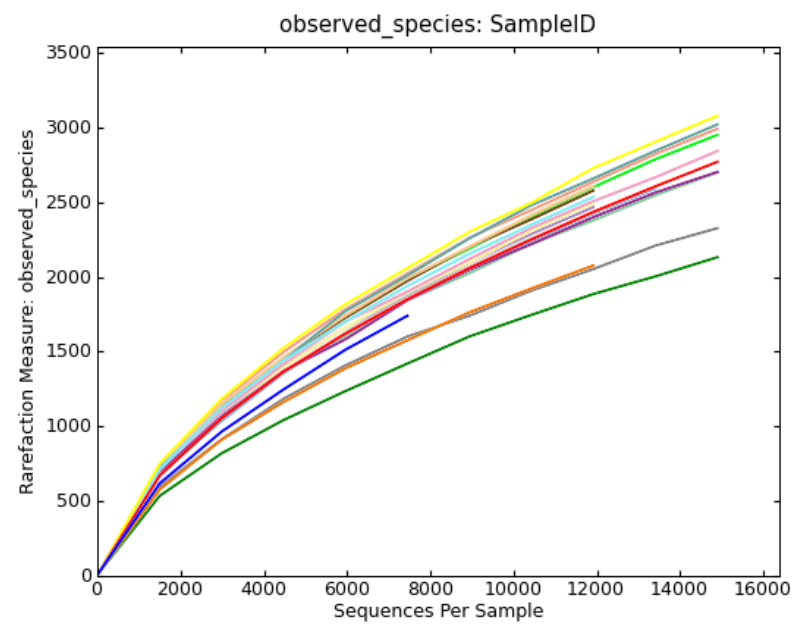

(A)

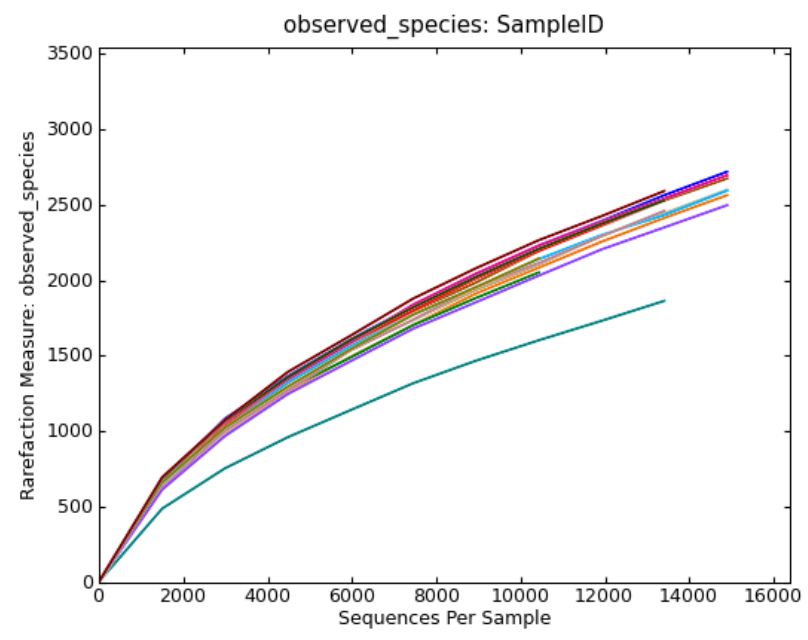

(B)

Figure 1. Rarefaction analysis of V4 reads of the 16S rRNA gene in fecal microbiota. Rarefaction curves were constructed at a $97 \%$ sequence similarity cut-off value; (A) Rarefaction analysis of Mongolian horses from 14 smples; (B) Rarefaction analysis of Thoroughbred horses from 19 smples.

into phylum, class, order, family and genera levels, which were $67,340,50,445,48,265,21,337$, and 6,806, respectively.

\section{Analysis of rarefaction curve and shannon index}

The rarefaction curve and the shannon index were analyzed for both horse populations and showed similar trends. The rarefaction analyses were carried out to determine whether all the OTUs present in the datasets had been sufficiently recovered. The individual rarefaction curves showed a similar pattern of reaching plateau but failing to reach a saturation phase (Figure 1). Curves for the

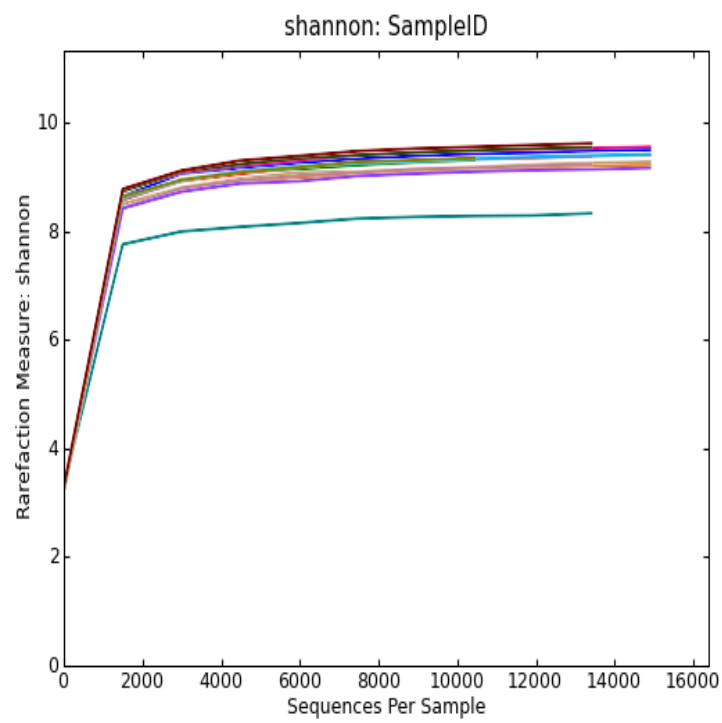

(A)
Shannon index plateau were at relatively low read numbers, but reached saturation (Figure 2). This suggests that a large number of unseen OTUs still existed in the original samples and more sequencing effort may be required to detect additional phylotypes. However, the saturation of microbial diversity is no longer changed.

\section{Analysis of alpha diversity}

The alpha diversity of fecal microbiota was estimated using Chao, ACE, Simpson, Shannon, and Coverage indices. The OTU richness estimated by Chao and ACE and average estimates of 2,143 (range $=1,841$ to $2,380, \mathrm{SD}=151)$ and

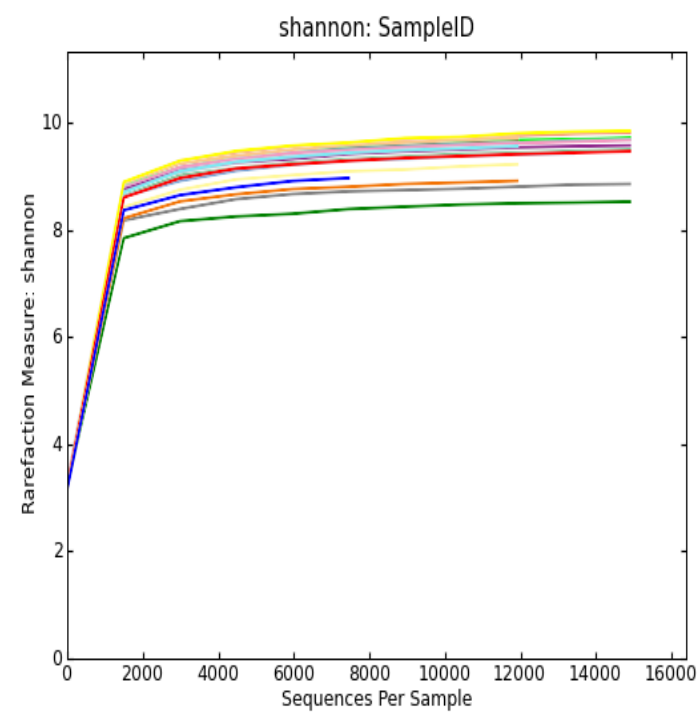

(B)

Figure 2. Shannon index of V4 reads of the 16S rRNA gene in fecal microbiota. Rarefaction curves were constructed at a $97 \%$ sequence similarity cut-off value; (A) Rarefaction analysis of Mongolian horses from 14 smples; (B) Rarefaction analysis of Thoroughbred horses from 19 smples. 
Table 1. Analysis of alpha diversity at $97 \%$ similarity

\begin{tabular}{lcccccc}
\hline Breed & OTUs & Chao & ACE & Simpson & Shannon & Coverage \\
\hline Mongolian horses & 37,057 & $2,143 \pm 151$ & $2,236 \pm 165$ & $0.0056 \pm 0.0020$ & $6.1107 \pm 0.2220$ & $0.9586 \pm 0.0086$ \\
Thoroughbred horses & 54,930 & $2,214 \pm 178$ & $2,260 \pm 149$ & $0.0058 \pm 0.0029$ & $6.1926 \pm 0.2500$ & $0.9595 \pm 0.0131$ \\
\hline
\end{tabular}

The richness estimators (ACE and Chao), diversity indices (Shannon and Simpson), and Coverage were calculated using the MOTHUR software.

2,214 (range $=1,817$ to $2486, \mathrm{SD}=178$ ) for Mongolian horses and 2,236 (range $=1,881$ to $2,624, \mathrm{SD}=165$ ) and 2,260 (range $=1,877$ to $2,474, \mathrm{SD}=149$ ) for Thoroughbred horses were obtained. Similarly, the Simpson's indexes were 0.0056 vs 0.0058 and the Shannon's indexes were 6.1107 vs 6.1926 (Table 1). We found that these five indices were higher in Thoroughbred horses than in Mongolian horses. However, the t-test revealed non-significant differences among the breeds. The analysis suggested that the diversity of gut bacterial community for the two breeds were basically the same. The coverage was $95.86 \%$ for

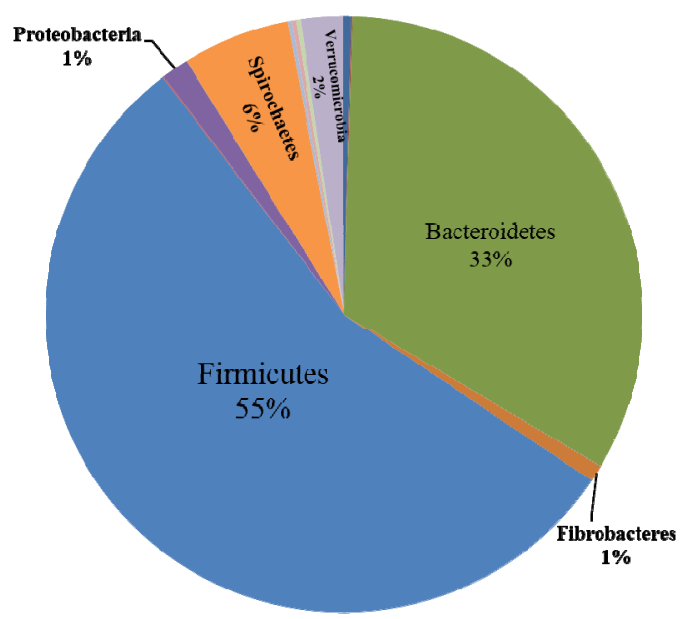

(A)

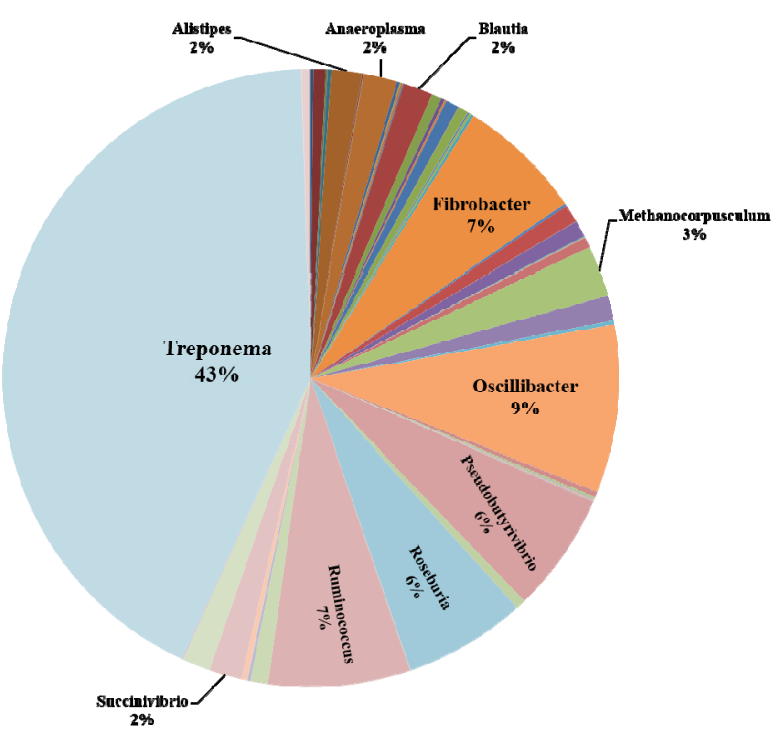

(C)
Mongolian horses and $95.95 \%$ for Thoroughbred horses $(\mathrm{p}>0.05)$ (Table 1).

\section{Analysis of community composition}

Sixteen phyla were detected in the gut microbiota of 33 horses. Six main phyla were found at varying proportions including 56\% and 53\% for Firmicutes, 33\% and 32\% for Bacteroidetes, $6 \%$ and 5\% for Spirochaete, $2 \%$ and $3 \%$ for Verrucomicrobia, $1 \%$ and $4 \%$ for Proteobacteria, and $1 \%$ and $1 \%$ for Fibrobacteres, in Mongolian horses and Thoroughbred horses, respectively (Figure 3A and 3B). The

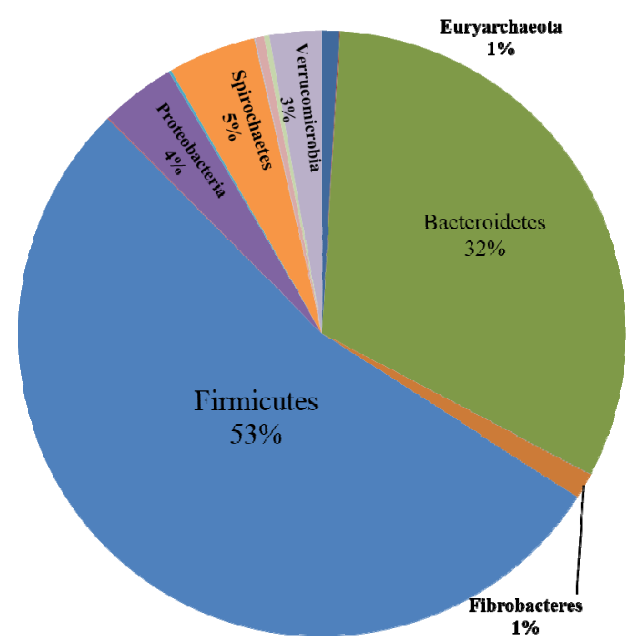

(B)

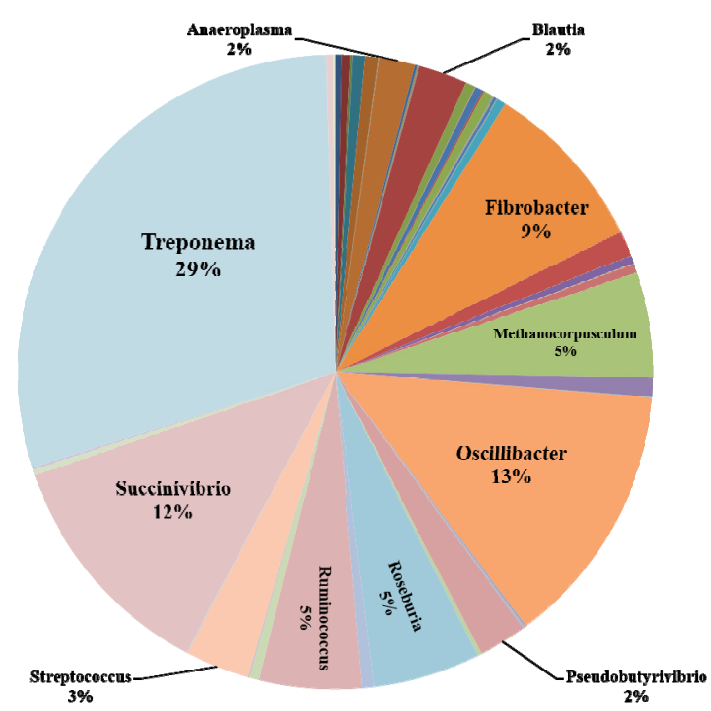

(D)

Figure 3. Distribution of the fecal microbiota composition. (A) Mongolian horses at the phylum level. (B) Thoroughbred horses at the phylum level. (C) Mongolian horses at genus level. (D) Thoroughbred horses at genus level. 
Firmicutes to Bacteroidetes $(\mathrm{F} / \mathrm{B})$ ratios were 1.67 for Mongolian horses and 1.68 for Thoroughbred horses. However, Euryarchaeota was $1 \%$ in Thoroughbred horses and less than $0.5 \%$ in Mongolian horses. Planctomycetes were not observed in Thoroughbred horses.

There were seventy-five genera. Proportion of the distribution was greater than $2 \%$ in 11 genera, but 10 genera were present in both breeds. Treponema (43\% vs 29\%, belong to Spirochaete), Ruminococcus (7\% vs 5\%, belong to Firmicutes), Roseburia (6\% vs 5\%, belong to Firmicutes), Pseudobutyrivibrio (6\% vs 2\%, belong to Firmicutes), and Anaeroplasma (3\% vs $2 \%$, belong to Tenericutes) were higher in Mongolian horses than in Thoroughbred horses. In contrast, Oscillibacter ( $9 \%$ vs 13\%, belong to Firmicutes), Fibrobacter ( $7 \%$ vs $9 \%$, belong to Fibrobacteres), Methanocorpusculum (3\% vs 5\%, belong to Euryarchaeota), and Succinivibrio ( $2 \%$ vs $12 \%$, belong to Proteobacteria) were lower in Mongolian horses than in Thoroughbred horses. The remaining Blautia (2\%, belong to Firmicutes) was identical (Figure 3C and 3D). Interestingly, Alistipes (belong to Bacteroidetes) of distribution proportion in Mongolian horses $(2 \%)$ was higher than in Thoroughbred horses (1\%). However, Streptococcus (belong to Firmicutes) was $35 \%$ in Thoroughbred horses and less than $1 \%$ in Mongolian horses.

\section{Analysis of relative abundance of fecal microbiota}

The relative abundance of 16 phyla and 75 genera were compared between the Mongolian and Thoroughbred horses. Of these 16 phyla, five phyla were significantly different $(p<0.05)$ between two breeds. Of these five phyla, Synergistetes and Planctomycetes were significantly higher in Mongolian horses than in Thoroughbred horses. Proteobacteria, TM7 and Chloroflexi were significantly lower in Mongolian horses than in Thoroughbred horses (Table 2).

Among 75 genera, 30 genera were significantly different $(\mathrm{p}<0.05)$ between Mongolian horses and Thoroughbred horses; of these 30 genera, Pseudobutyrivibrio, Synergistes, Pseudomonas, Phascolarctobacterium, Anaerobiospirillum, Helicobacter, Ruminococcus, Parabacteroides, Campylobacter, Butyricimonas, Roseomonas, Treponema, Polynucleobacter, Schwartzia, Parasutterella, Odoribacter, Turicibacter, and Barnesiella were significantly higher in Mongolian horses than in Thoroughbred horses. Succinivibrio, Ruminobacter, Faecalibacterium, Streptococcus, Blautia, Paludibacter, Akkermansia, Weissella, Oscillibacter, Subdoligranulum, Anaerovorax, and Doreawere significantly lower in Mongolian horses than in Thoroughbred horses (Table 3).

Among these 30 genera, we observed 13 genera belonging to Firmicutes, 9 genera belonging to Proteobacteria, and 5 genera belonging to Bacteroidetes. Additional abundant genera included Treponema (belonging to Spirochaete), Ruminococcus (belonging to Firmicutes), Pseudobutyrivibrio (belonging to Firmicutes), Oscillibacter (belonging to Firmicutes), Succinivibrio (belonging to Proteobacteria), and Blautia (belonging to Firmicutes) (Table 3).

\section{DISCUSSION}

This is the first work which reports the composition of gut microbiota in the Mongolian horses. Previous studies used various techniques to study the gut microbiota composition in horses. Costa et al. (2012) reported Firmicutes as the predominant taxa $(39.5 \%$ to $72.6 \%)$ followed by Bacteroidetes (8.9\% to $21.3 \%$ ) in healthy horses populations (Thoroughbred, mixed-bread pony, mixed-bread, and Quarter Horse) (Costa et al., 2012). Shepherd et al. (2012) found that the bacterial communities were dominated by Firmicutes (43\%), followed by Verrucomicrobia (4.1\%), Proteobacteria (3.8\%), and Bacteroidetes (3.7\%) from horses fed cool-season grass hay (Shepherd et al., 2012). O'Donnell et al. (2013) revealed that the dominance of Firmicutes and Bacterioidetes phyla in the fecal microbiome of Irish Thoroughbred race horses (O'Donnell et al., 2013). In the current study, we detected that microbiota dominated by Firmicutes (56\% and 53\%) and Bacteroidetes (33\% and 32\%) in the Mongolian and Thoroughbred horses respectively. This indicates that the proportion of Firmicutes was similar in the horse gut microbiomes. The Firmicutes and Bacteroidetes phyla predominated in donkey, human, pig and beef cattle feces suggesting the ecological and functional importance of this group within the gut across species (Larsen et al., 2010; Lamendella et al., 2011; Shanks et al., 2011; Liu et al., 2014). The predominance of Firmicutes may be related to

Table 2. Breed comparisons for the relative abundance of fecal microbiota at the phylum level

\begin{tabular}{lcc}
\hline Phylum & $\begin{array}{c}\text { Relative fold change } \\
\text { Log2 (Mongolian horses/Thoroughbred horses) }\end{array}$ & $\mathrm{p}$ value \\
\hline Synergistetes & 2.1331 & $1.2786 \mathrm{E}-06^{* *}$ \\
Proteobacteria & -1.4123 & $8.3643 \mathrm{E}-06^{* *}$ \\
Planctomycetes & 13.3426 & $0.0142^{*}$ \\
TM7 & -0.9390 & $0.0350^{*}$ \\
Chloroflexi & -1.9481 & $0.0482^{*}$ \\
\hline
\end{tabular}


Table 3. Breed comparisons for the relative abundance of fecal microbiota at the genus level

\begin{tabular}{|c|c|c|c|}
\hline Phylum & Genus & $\begin{array}{c}\text { Relative fold change } \\
\text { Log2 (Mongolian horses/Thoroughbred horses) }\end{array}$ & $\mathrm{p}$ value \\
\hline \multirow[t]{13}{*}{ Firmicutes } & Faecalibacterium & -1.8926 & $7.8789 \mathrm{E}-07^{* *}$ \\
\hline & Streptococcus & -3.6901 & $9.9651 \mathrm{E}-07 * *$ \\
\hline & Pseudobutyrivibrio & 1.1544 & $1.2651 \mathrm{E}-06^{* *}$ \\
\hline & Blautia & -0.8724 & $5.7925 \mathrm{E}-05^{* *}$ \\
\hline & Weissella & -2.9881 & $1.3100 \mathrm{E}-03 * *$ \\
\hline & Phascolarctobacterium & 12.7212 & $2.0149 \mathrm{E}-03 * *$ \\
\hline & Oscillibacter & -0.8226 & $6.6909 \mathrm{E}-03 * *$ \\
\hline & Subdoligranulum & -2.6774 & $0.0101 *$ \\
\hline & Ruminococcus & 0.3148 & $0.0110 *$ \\
\hline & Anaerovorax & -12.0620 & $0.0214 *$ \\
\hline & Dorea & -1.3856 & $0.0251 *$ \\
\hline & Schwartzia & 0.7161 & $0.0443 *$ \\
\hline & Turicibacter & 12.2525 & $0.0477 *$ \\
\hline \multirow[t]{5}{*}{ Bacteroidetes } & Paludibacter & -3.7011 & $4.0086 \mathrm{E}-04 * *$ \\
\hline & Parabacteroides & 3.1402 & $0.0154 *$ \\
\hline & Butyricimonas & 5.3054 & $0.0250 *$ \\
\hline & Odoribacter & 2.8890 & $0.0465 *$ \\
\hline & Barnesiella & 3.6558 & $0.0499 *$ \\
\hline \multirow[t]{9}{*}{ Proteobacteria } & Succinivibrio & -3.0063 & $1.2643 \mathrm{E}-09 * *$ \\
\hline & Ruminobacter & -4.3546 & $3.5337 \mathrm{E}-07 * *$ \\
\hline & Pseudomonas & 1.1275 & $1.8753 \mathrm{E}-04 * *$ \\
\hline & Anaerobiospirillum & 13.6460 & $2.9365 \mathrm{E}-03^{* *}$ \\
\hline & Helicobacter & 5.3204 & $4.0298 \mathrm{E}-03 * *$ \\
\hline & Campylobacter & 1.7569 & $0.0216^{*}$ \\
\hline & Roseomonas & 12.0733 & $0.0282 *$ \\
\hline & Polynucleobacter & 3.9527 & $0.0427^{*}$ \\
\hline & Parasutterella & 3.7548 & $0.0461^{*}$ \\
\hline Synergistetes & Synergistes & 2.0926 & $2.4499 \mathrm{E}-06^{* *}$ \\
\hline Verrucomicrobia & Akkermansia & -1.9840 & $1.0943 \mathrm{E}-03 * *$ \\
\hline Spirochaetes & Treponema & 0.3388 & $0.0295^{*}$ \\
\hline
\end{tabular}

$* \mathrm{p}<0.05 ; * * \mathrm{p}<0.01$.

the anatomical physiology and feeding habits of this species which ingests mainly insoluble fiberand uses the cecum and large colon as the main sites for fermentation (Costa et al., 2012). However, in this study, two horse breeds had different feeding habits and environment, nevertheless, showed the same dominant microbial community $R$ (Firmicutes). Some reports indicated that the abundances of Bacteroidetes and Firmicutescan were highly biased in cases of obesity (Ley et al., 2006), diabetes (Sasaki et al., 2013), and cardiovascular risk factors (Manco et al., 2010). We analyzed the Firmicutes to Bacteroidetes (F/B) ratios that were 1.67 and 1.68 in Mongolian horses and Thoroughbred horses. This ratio was lower than for Irish Thoroughbred race horses (>2:1) (Costa et al., 2012).

Some studies showed that dietary manipulation can also have an impact on the composition of gut microbiota (Costa and Weese, 2012). This is mainly because the horse depends upon microbial fermentation to digest plant structural carbohydrates (Hintz and Cymbaluk, 1994). Later, horses received supplementation with concentrate had 10 times more lactic acid bacteria than horses receiving a forage-only diet (Willing et al., 2009). High concentrate diet could increase abundance of Clostridiaceae cluster XIVa and Bacteroidetes assemblage, anddecreased abundance of Ruminococcaceae, comparing with hay-based diet (Daly et al., 2012). In this study, Mongolian and Thoroughbred horses lived in a different environment and had different lifestyle. Mongolian horses were on the prairie and have free access to feed the grass which is not available for Thoroughbred horses. The later population gets concentrate feeds and grasses in scheduled time. It is worth noting that Proteobacteria was significantly different in two breeds ( $1 \%$ and 4\%, Mongolian horses and Thoroughbred horses respectively). Proteobacteria included many pathogenic bacteria, such as salmonella, V. cholera, Helicobacter pylori, etc. At the genus level, Streptococcus was $3 \%$ for Thoroughbred horses and less than $1 \%$ for Mongolian horses, and was significantly different in two breeds. 
Streptococcus also included pathogenic bacteria (e.g. Streptococcus pneumoniae, Streptococcus pyogenes, Streptococcus agalactiae) (Chao et al., 2014; Cole et al., 2014). Therefore, these data might be of an important factor that caused Thoroughbred horses to be more susceptible to diseases than Mongolian horses. This needs to be confirmed further. Meanwhile, we discovered 5 phyla and 30 genera were significantly different between Mongolian and Thoroughbred horses, which suggested that breeds, feeding and environmentas factors may influence the composition of gut microbiota.

At the genus level, the proportional distribution of the different species were detected in main genera. Ruminococcus and Fibrobacter were strong fiber degrading bacteria. The analysis of relative abundance difference needs further study. We observed Ruminococcus was significantly different among the two breeds. This result was consistent with the characteristics of grazing that reflects the two breeds have different fiber digesting. Others, Treponema, Oscillibacter, Pseudobutyrivibrio, and Succinivibrio were also significantly different among the two breeds. Nevertheless, Ruminococcus, Pseudobutyrivibrio, and Succinivibrio existed in rumen (Mohammed et al., 2014; Gharechahi et al., 2015). Schoster et al. indicated the highest similarity was obtained when feces were compared to the cecum (Schoster et al., 2013). This further evidenced that the cecum of horses was very important. The beneficial microflora contains Lactobacillus, Bifidobacterium, and others. In the current study, we found that Lactobacillus counts in Mongolian horses $(0.86 \%)$ were higher than in Thoroughbred horses $(0.42 \%)$, ther no significant difference observed. Very few Bifidobacterium of beneficial bacterium obtained in the two breeds.

\section{CONCLUSION}

In this study, we first investigated gut microbiota of Mongolian horses and compared the fecal microbiota of Mongolian horses and Thoroughbred horses. The two breeds had similar bacterial community compositions which belonged to 16 phyla and 75 genera. The most abundant phylum were Firmicutes and Bacteroidetes. Noteworthy, Proteobacteria (1\% and 4\%, Mongolian horses and Thoroughbred horses), and Streptococcus $(3 \%$ in Thoroughbreds, less than $1 \%$ in Mongolian horses) were significantly different among the two breeds.

\section{CONFLICT OF INTEREST}

We certify that there is no conflict of interest with any financial organization regarding the material discussed in the manuscript.

\section{ACKNOWLEDGMENTS}

This work was supported by Ministry of Science and Technology of the People's Republic of China specific scientific and technological cooperation with Russia (2011DFR30860), Inner Mongolia key laboratory project (20130902), Research Program of science and technology at Universities of Inner Mongolia Autonomous Region (NJZY0701), Supported by Natural Science Foundation of Inner Mongolia Autonomous Region of china (2015BS0316). We thank Shanghai Personal Biotechnology Limited Company for the analysis of data. We thank Dr. Ming Du in China and Dr. Mark Stanislaw Goettel in US for proofreading the manuscript.

\section{REFERENCES}

Blackmore, T. M., A. Dugdale, C. M. Argo, G. Curtis, E. Pinloche, P. A. Harris, H. J. Worgan, S. E. Girdwood, K. Dougal, C. J. Newbold, and N. R. McEwan. 2013. Strong stability and host specific bacterial community in faeces of ponies. PloS One 8: e75079.

Chao, Y., L. R. Marks, M. M. Pettigrew, and A. P. Hakansson. 2014. Streptococcus pneumoniae biofilm formation and dispersion during colonization and disease. Front. Cell. Infect. Microbiol. 4:194.

Cole, J., J. Aberdein, J. Jubrail, and D. H. Dockrell. 2014. The role of macrophages in the innate immune response to Streptococcus pneumoniae and Staphylococcus aureus: Mechanisms and contrasts. Adv. Microb. Physiol. 65:125-202.

Costa, M. C., L. G. Arroyo, E. Allen-Vercoe, H. R. Stampfli, P. T. Kim, A. Sturgeon, and J. S. Weese. 2012. Comparison of the fecal microbiota of healthy horses and horses with colitis by high throughput sequencing of the V3-V5 region of the $16 \mathrm{~S}$ rRNA gene. PloS one 7:e41484.

Costa, M. C. and J. S. Weese. 2012. The equine intestinal microbiome. Anim. Health Res. Rev. Conference of Research Workers in Animal Diseases 13:121-128.

Daly, K., C. J. Proudman, S. H. Duncan, H. J. Flint, J. Dyer, and S. P. Shirazi-Beechey. 2012. Alterations in microbiota and fermentation products in equine large intestine in response to dietary variation and intestinal disease. Br. J. Nutr. 107:989995.

Dougal, K., G. de la Fuente, P. A. Harris, S. E. Girdwood, E. Pinloche, R. J. Geor, B. D. Nielsen, H. C. Schott 2nd, S. Elzinga, and C. J. Newbold. 2014. Characterisation of the faecal bacterial community in adult and elderly horses fed a high fibre, high oil or high starch diet using 454 pyrosequencing. PloS one 9:e87424.

Dougal, K., P. A. Harris, A. Edwards, J. A. Pachebat, T. M. Blackmore, H. J. Worgan, and C. J. Newbold. 2012. A comparison of the microbiome and the metabolome of different regions of the equine hindgut. FEMS Microbiol. Ecol. 82:642-652.

Gharechahi, J., H. S. Zahiri, K. A. Noghabi, and G. H. Salekdeh. 2015. In-depth diversity analysis of the bacterial community resident in the camel rumen. Syst. Appl. Microbiol. 38:67-76. 
Gustafsson, A., V. Baverud, A. Franklin, A. Gunnarsson, G. Ogren, and C. Ingvast-Larsson. 1999. Repeated administration of trimethoprim/sulfadiazine in the horse--pharmacokinetics, plasma protein binding and influence on the intestinal microflora. J. Vet. Pharmacol. Ther. 22:20-26.

Hintz, H. F. and N. F. Cymbaluk. 1994. Nutrition of the horse. Ann. Rev. Nutr. 14:243-267.

Lamendella, R., J. W. Domingo, S. Ghosh, J. Martinson, and D. B. Oerther. 2011. Comparative fecal metagenomics unveils unique functional capacity of the swine gut. BMC Microbiol. 11:103.

Larsen, N., F. K. Vogensen, F. W. van den Berg, D. S. Nielsen, A. S. Andreasen, B. K. Pedersen, W. A. Al-Soud, S. J. Sorensen, L. H. Hansen, and M. Jakobsen. 2010. Gut microbiota in human adults with type 2 diabetes differs from non-diabetic adults. PloS one 5:e9085.

Ley, R. E., M. Hamady, C. Lozupone, P. J. Turnbaugh, R. R. Ramey, J. S. Bircher, M. L. Schlegel, T. A. Tucker, M. D. Schrenzel, R. Knight, and J. I. Gordon. 2008. Evolution of mammals and their gut microbes. Science 320:1647-1651.

Ley, R. E., P. J. Turnbaugh, S. Klein, and J. I. Gordon. 2006. Microbial ecology: Human gut microbes associated with obesity. Nature 444:1022-1023.

Liu, X., H. Fan, X. Ding, Z. Hong, Y. Nei, Z. Liu, G. Li, and H. Guo. 2014. Analysis of the gut microbiota by high-throughput sequencing of the $\mathrm{V}_{5}-\mathrm{V}_{6}$ regions of the $16 \mathrm{~S}$ rRNA gene in donkey. Curr. Microbiol. 68:657-662.

Manco, M., L. Putignani, and G. F. Bottazzo. 2010. Gut microbiota, lipopolysaccharides, and innate immunity in the pathogenesis of obesity and cardiovascular risk. Endocr. Rev. 31:817-844.

Mohammed, R., G. E. Brink, D. M. Stevenson, A. P. Neumann, K. A. Beauchemin, G. Suen, and P. J. Weimer. 2014. Bacterial communities in the rumen of Holstein heifers differ when fed orchardgrass as pasture vs. hay. Front. Microbiol. 5:689.
O'Donnell, M. M., H. M. Harris, I. B. Jeffery, M. J. Claesson, B. Younge, P. W. O'Toole, and R. P. Ross. 2013. The core faecal bacterial microbiome of Irish Thoroughbred racehorses. Lett. Appl. Microbiol. 57:492-501.

Obitsu, T., H. Hata, and K. Taniguchi. 2015. Nitrogen digestion and urea recycling in Hokkaido native horses fed hay-based diets. Anim. Sci. J. 86:159-165.

Sasaki, M., N. Ogasawara, Y. Funaki, M. Mizuno, A. Iida, C. Goto, S. Koikeda, K. Kasugai, and T. Joh. 2013. Transglucosidase improves the gut microbiota profile of type 2 diabetes mellitus patients: A randomized double-blind, placebo-controlled study. BMC Gastroenterol. 13:81.

Schoster, A., L. G. Arroyo, H. R. Staempfli, and J. S. Weese. 2013. Comparison of microbial populations in the small intestine, large intestine and feces of healthy horses using terminal restriction fragment length polymorphism. BMC Res. Notes 6: 91.

Shanks, O. C., C. A. Kelty, S. Archibeque, M. Jenkins, R. J. Newton, S. L. McLellan, S. M. Huse, and M. L. Sogin. 2011. Community structures of fecal bacteria in cattle from different animal feeding operations. Appl. Environ. Microbiol. 77: 2992-3001.

Shepherd, M. L., W. S. Swecker, Jr., R. V. Jensen, and M. A. Ponder. 2012. Characterization of the fecal bacteria communities of forage-fed horses by pyrosequencing of $16 \mathrm{~S}$ rRNA V4 gene amplicons. FEMS Microbiol. Lett. 326:62-68.

Steelman, S. M., B. P. Chowdhary, S. Dowd, J. Suchodolski, and J. E. Janecka. 2012. Pyrosequencing of 16S rRNA genes in fecal samples reveals high diversity of hindgut microflora in horses and potential links to chronic laminitis. BMC Vet. Res. 8:231.

Willing, B., A. Voros, S. Roos, C. Jones, A. Jansson, and J. E. Lindberg. 2009. Changes in faecal bacteria associated with concentrate and forage-only diets fed to horses in training. Equine Vet. J. 41:908-914. 